\title{
Sine Wave Inverter Bipolar SPWM using Analog Triggering Circuit
}

\author{
Catur Rakhmad Handoko \\ Ship Electrical Departement \\ Shipbuilding Institut of Polytechnic Surabaya \\ Surabaya, Indonesia
}

\begin{abstract}
An inverter is needed when an electric load requires an $\mathrm{AC}$ power supply while the available one is a DC power supply. The quality of an inverter, one of which is determined by, among others, by the output waveform, whether a square wave, modified sinuses or pure sinuses. Among the three, pure sine inverters require more complicated conversion stages. One technique is bipolar Sinusoidal Pulse Width Modulation (SPWM). In this simulation, an low cost analog trigger signal is generated for the semiconductor switching process using a signal carrier with a frequency of $800 \mathrm{~Hz}$ which is adjusted to the HF Transformer. Furthermore, the modulation results are filtered with a Low Pass LC filter to produce a sine waveform output at 220 Volt and $50 \mathrm{~Hz}$.
\end{abstract}

Keywords-Inverter, Sinusoidal Pulse Width Modulation, Analog Circuit

\section{INTRODUCTION}

Many electricity sources that can not be separated from the use of current inverter include: UPS (Unteruptable Power System) which is a backup power source that comes from batteries that have a direct voltage (DC) and then converted through an inverter to alternating voltage (AC) so that it can replace the electrical energy from the main power supply (PLN).

The inverter which is used as a power supply provides benefits as the main power supply (PLN), which generates a constant voltage / current and a constant frequency even if there is a change in load. However, a drawback arises, namely that this inverter always produces large harmonics, compared to the source of the generator (PLN) that does not have harmonics because the voltage / current is in the form of pure sine (Pure Sine Wave). This harmonic affects the quality of energy (Power System Quality) produced by the inverter and will disrupt and simultaneously shortene the life time of the load. For example, if the inverter is used to drive a motor in an altered frequency condition, the motor will emit noise because of the harmonics contained in the input voltage. The result can be unstable heat and torque at low speeds. Even the harmonics produced by the inverter which are also accompanied by noise, ripple, transient can cause load operations to be inefficient and can damage the device.

The DC power source that is the input of the inverter can be a battery or rectifier output circuit which is part of the AC AC (DC Link) conversion system. The output of a single phase inverter is usually used to supply AC $220 \mathrm{~V} 50 \mathrm{~Hz}$ loads such as lights, single phase induction motors and other loads with $220 \mathrm{~V}$ input..

\section{SINE WAVE INVERTER}

Based on the waveform, the output of an inverter is divided into 3 types, namely square wave, modified sinus and pure sinus as illustrated in Figure 1. Of the three waveforms, sine wave inverters have the best quality, especially from the level of harmonic distortion (\% THD ) The sine inverter is the lowest.

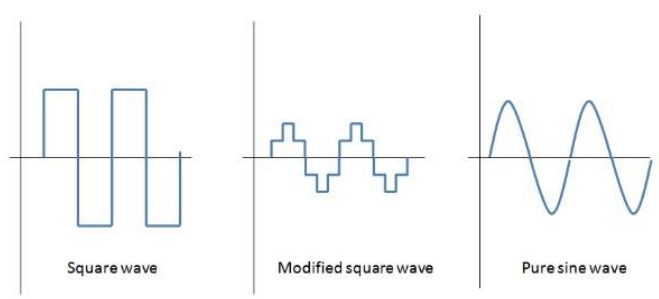

Fig 1 Various Waveform of an inverter

All types of inverters basically have the same circuit base, namely the fullbridge wave band circuit as shown in figure 2 . The square waveform, the simplest, is formed when switches $\mathrm{Q}_{1}, \mathrm{Q}_{3}$ are temporarily $\mathrm{Q}_{2}, \mathrm{Q}_{4} \mathrm{OFF}$, so alternately. This combination will cause the current to flow to the load according to the arrow. This combination runs for half the period of the frequency of the desired ac voltage. For example, the desired frequency of output is $\mathrm{f}=50 \mathrm{~Hz}$ or $\mathrm{T}=$ $1 / \mathrm{f}=20 \mathrm{~ms}$, so $\mathrm{Q}_{1}$ and $\mathrm{Q}_{4}$ operate for 10 milliseconds. This means that the positive current will flow to the load for 10 $\mathrm{ms}$. The next combination deactivates $\mathrm{Q}_{1}$ and $\mathrm{Q}_{4}$ and turns on $\mathrm{Q}_{2}$ and $\mathrm{Q}_{3}$. The $\mathrm{Q}_{2}$ and $\mathrm{Q}_{3}$ switches also operate for $10 \mathrm{~ms}$, with a negative current in the load. Continuously, so that a square wave will form, with a period of $20 \mathrm{~ms}$ or $50 \mathrm{~Hz}$

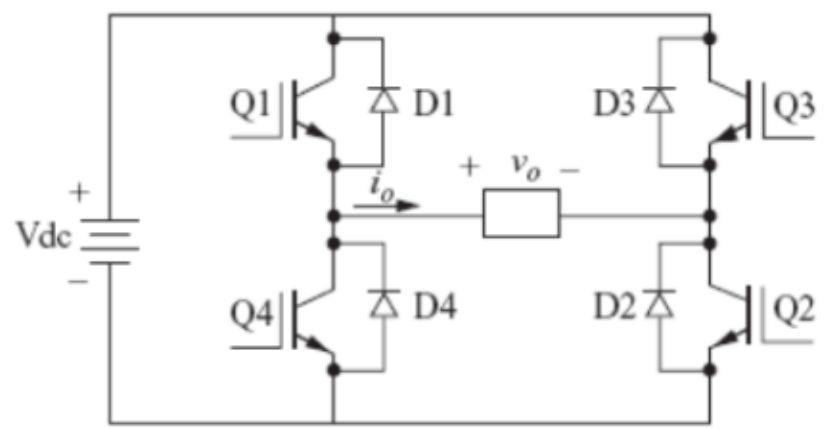

Fig 2 Basic circuit of an inverter 
The next challenge is how to form a sine wave from this square wave. Although it can be used for certain AC loads such as lighting, but is a square waveform, it is inefficient and contains a high harmonic distortion. The following is a simple analysis of harmonics on a square wave and how to convert it to a sinus.

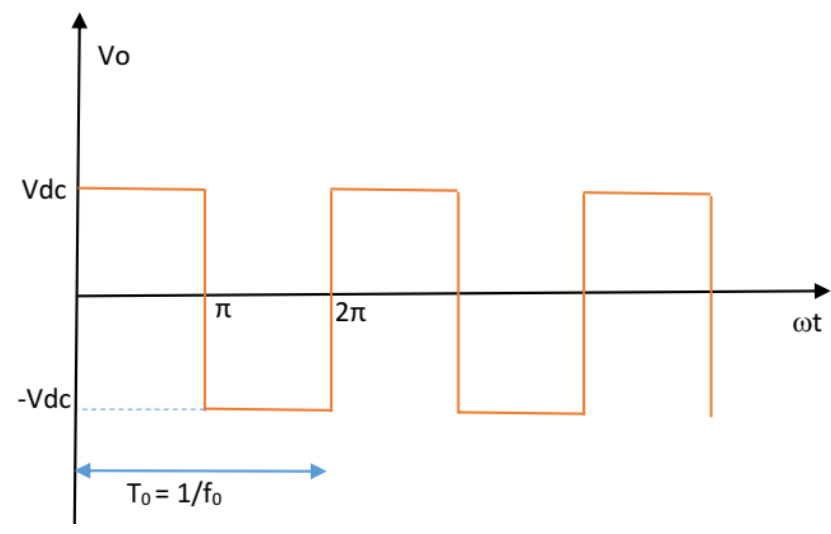

Fig 3 Square waveform

The square wave above, as a function of time can be formulated as follows:

$$
\begin{aligned}
\mathrm{V}(\omega \mathrm{t}) & =\mathrm{V}_{\mathrm{dc}} \text { for } 0 \leq \omega \mathrm{t} \leq \pi \\
& =\mathrm{V}_{\mathrm{dc}} \text { for } \pi \leq \omega \mathrm{t} \leq 2 \pi
\end{aligned}
$$

And the fourier equition are :

$$
\begin{gathered}
a_{0}=0 \\
a_{n}=0 \\
b_{n}=\frac{4 V d e}{n \pi} \quad \text { for } n=1,3,5,7, \ldots
\end{gathered}
$$

And it is obtained that

$$
V \omega t=\frac{4 V d c}{\pi} \sin \omega t+\frac{4 V d e}{2 \pi} \sin \omega t+\frac{4 V d e}{5 \pi} \sin \omega t+\ldots(5)
$$

This means that the square wave with the peak Vdc is the sum of :

$$
\begin{aligned}
& 50 \mathrm{~Hz} \text { sine wave with amplitude } \frac{4 V d c}{\pi} \\
& 150 \mathrm{~Hz} \text { sine wave with amplitude } \frac{4 V d c}{a \pi} \\
& 250 \mathrm{~Hz} \text { sine wave with amplitude } \frac{4 V d c}{5 \pi}
\end{aligned}
$$

Then by using the principle of series resonance, the unwanted frequencies can be filtered by throwing them into a neutral wire. For that purpose, a series, serial LC, will be tuned for frequencies of $150 \mathrm{~Hz}, 250 \mathrm{~Hz}, 350 \mathrm{~Hz}$ and the next respectively. Each of these filters is installed parallel to the load. The LC circuit impedance will be zero at those frequencies, so that it will be channeled to the ground. Meanwhile, for low frequencies, the fundamental frequency of $50 \mathrm{~Hz}$ will still flow to the load. Output waveforms become sine, although not perfect.

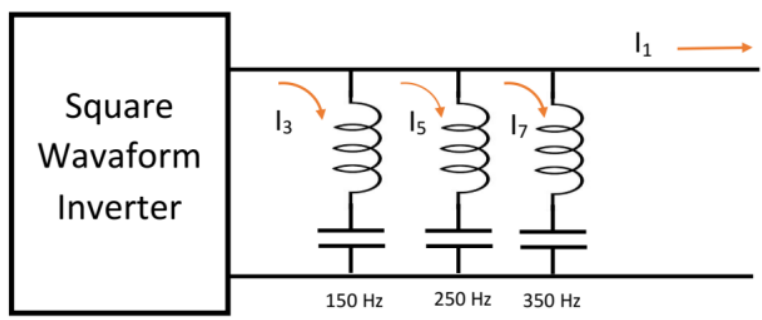

Fig 4. LC Filter in Square waveform inverter

Another way that can be used to make a sine wave from a square wave is a low pass filter like in figure 5. The $\mathrm{L}$ and $\mathrm{C}$ values are calculated in such a way that at the cut-off frequency the $X_{L}$ inductive impedance value is very low and the capacitive impedance of $\mathrm{X}_{\mathrm{C}}$ is very high, so the current flows to load R. Conversely, at a high frequency above the cut-off, the inductive impedance is very high and capacitive impedance is very small.

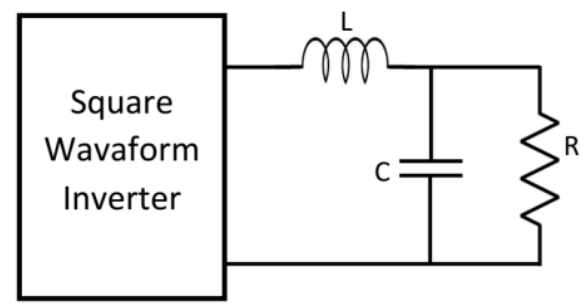

Fig 5. Low Pass Filter in Square waveform inverter

Meanwhile other waveforms are produced by further adjusting the semiconductor switching process in the existing basic sequence. One strategy for generating sine waves from the basic sequence above is the Sinusoidal Pulse Width Modulation (SPWM) technique. A $50 \mathrm{~Hz}$ reference sine wave is modulated along with a high frequency carrier wave. From this modulation, the trigger pattern is generated for the power transistors in the above sequence. The result is a high frequency wave that will be induced through a Step-up High Frequency Transformer to the secondary side of the transformer. Next, a low pass LC filter is installed which will pass a low frequency signal, which is the corresponding signal with a reference frequency of $50 \mathrm{~Hz}$.

\section{BIPOLAR SINUSOIDAL PWM}

\section{A. SPWM wave generator}

A Sinusoidal wave generator circuit Pulse Width Modulation (SPWM) consists of several components namely a $50 \mathrm{~Hz}$ sinus reference wave generator $\left(\mathrm{V}_{\text {sinref }}\right)$, a triangle wave generator $\left(\mathrm{V}_{\text {carrier }}\right)$, a comparator, a logic gate and gate buffers as shown in figure 3 . 


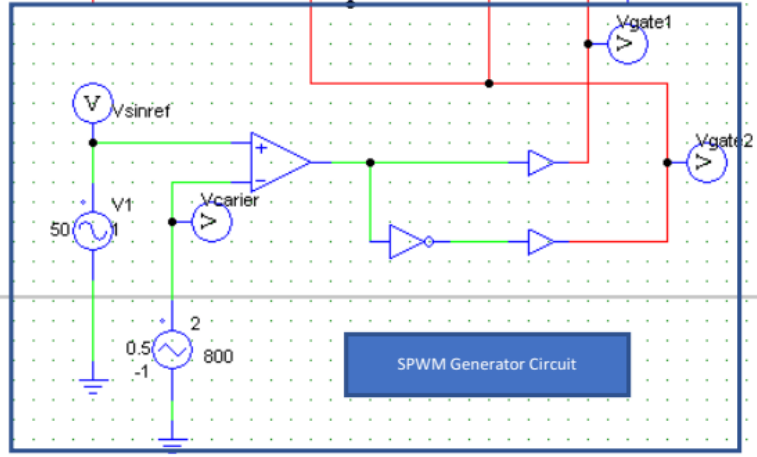

Fig 6 The Circuit of SPWM Generator

\section{B. SPWM Bipolar Signal Generation}

The comparator compares the two inputs, namely Vsinref and Vcarrier which will generate variations "high" and "low" on the comparator output. When the triangle signal is on the outside Vdc of the sine signal it will form an output signal to high. And when the triangle signal is in the sine signal it will form an output signal to be low. The comparator output is pulses with varying widths that are proportional to the reference wave amplitude $\left(\mathrm{V}_{\text {sinref }}\right)$ and the carrier wave density $\left(\mathrm{V}_{\text {carrier }}\right)$. This process is shown in Figure 4. This output is then processed using logic gates and buffers before being fed to semiconductor switches in the base array of inverters.
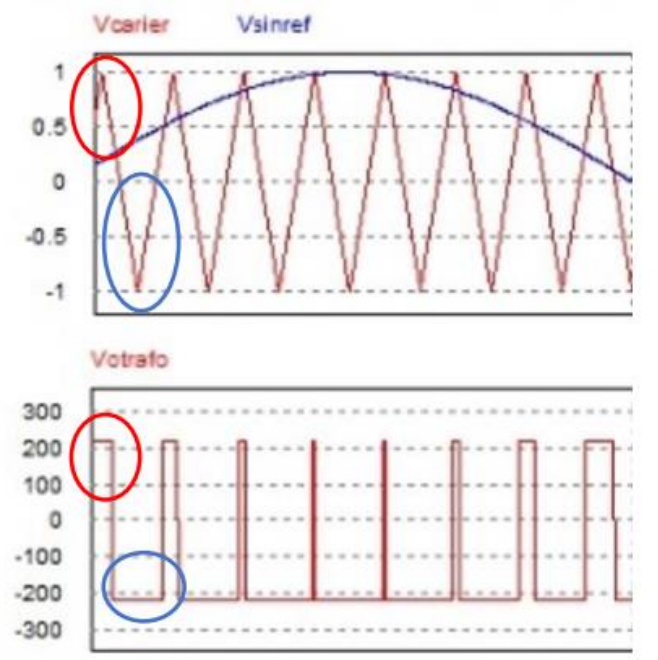

Fig 7 Bipolar SPWM signal generation

\section{Step-up High Frequency Transformer}

In the fullbridge inverter circuit, the semiconductor switches $\mathrm{Q}_{1}$ and $\mathrm{Q}_{4}$ will then be $\mathrm{ON}$ and OFF together, while the switches $\mathrm{Q}_{2}$ and $\mathrm{Q}_{3}$ will work in opposition to switches $\mathrm{Q}_{1}$ and $\mathrm{Q}_{4}$, according to the pattern formed from the SPWM bipolar wave generator circuit. The result is a combination of a high frequency wave with a low amplitude. To increase the amplitude that is close to the output target of 220 volts, this wave is passed a high frequency transformer with a winding ratio that corresponds to the number of turns of the primary and secondary transformer coils, which are directly proportional to the voltage. This simulation uses a carrier wave frequency of $800 \mathrm{~Hz}$.

\section{Filtering the output}

To produce the output wave as expected, a filter is used, which will return the SPWM wave with the amplitude of the voltage and frequency that is approaching the target. This filter is basically a low pass filter, which will only pass waves with low frequencies. In this simulation, the $\mathrm{L}$ and $\mathrm{C}$ values for the LC filter are obtained at $3 \mathrm{mH}$ and $1 \mathrm{uF}$ with a cut-off tuned resonance frequency of $92 \mathrm{~Hz}$. These values, in simulation, produce the best form of output.

\section{PSIM SIMULATION}

The following is an explanation of inverter circuit simulations using the bipolar SPWM method. This simulation uses PSIM software.

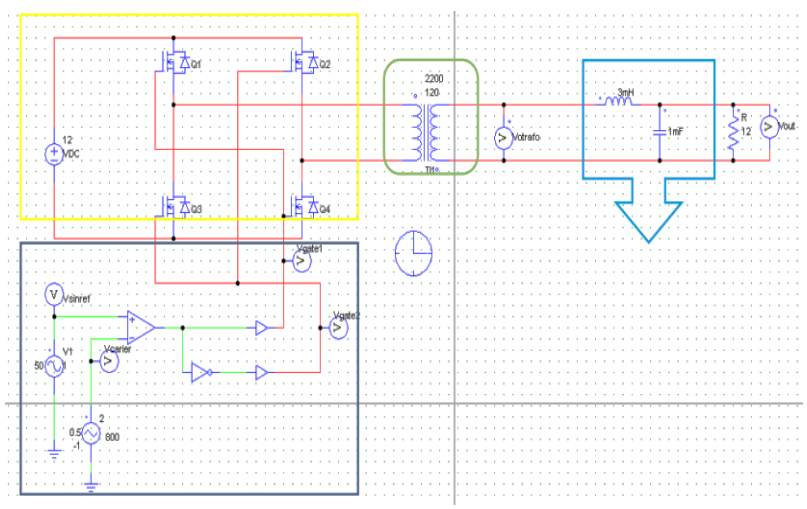

Fig 8 PSIM Bipolar SPWM Inverter System

The DC 12 Volt input signal will be chopped by switches $\mathrm{Q}_{1}, \mathrm{Q}_{3}$ and $\mathrm{Q}_{2}, \mathrm{Q}_{4}$, which will be ON-OFF according to the pattern of pulse width formed from the ranking of the SPWM bipolar wave generator. Figure 9 shows the output form of the SPWM generation circuit. The lower signal is the negation of the upper signal. Upper Side Signal as feed for mosfet $\mathrm{Q}_{1}$ and $\mathrm{Q}_{4}$. While the lower signal is for mosfet feeds $\mathrm{Q}_{2}$ and $\mathrm{Q}_{3}$.

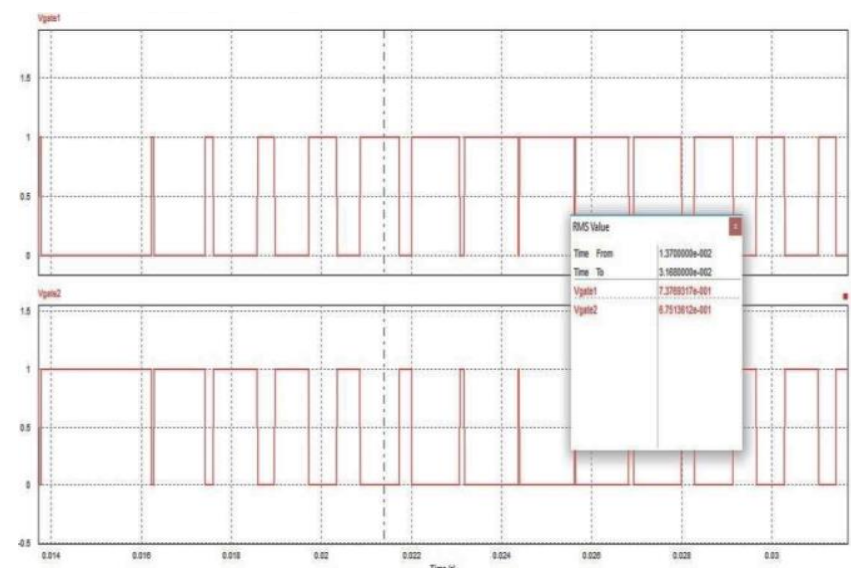

Fig 9 The Output of SPWM generation circuit

The next step is to amplify the voltage using step-up high frequency transformers. This transformer finally produces an SPWM wave with an amplitude of 219.7 volts which has approached the target voltage of 220 volts. As shown in figure 10 . 


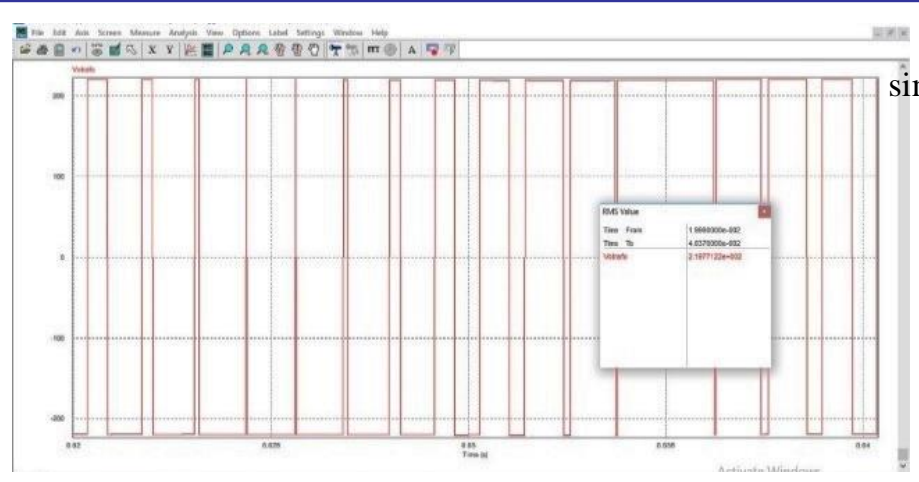

Fig 10. The output of HF Transformer

And finally, in figure 11, the output of HF transformers is filtered using the Low Pass LC Filter, tuned at a cut-off frequency of $92 \mathrm{~Hz}$. This value is the best value from the results of changing the $\mathrm{LC}$ value in the simulation by paying attention to the target frequency of $50 \mathrm{~Hz}$.

In determining the value of $\mathrm{L}$ and $\mathrm{C}$, also note whether the value of available capacitors on the market. While the value of $\mathrm{L}$ is calculated later after $\mathrm{C}$ with the formula for resonance frequency.

$$
f_{\text {res }}=\frac{1}{2 \pi \sqrt{L C}}
$$

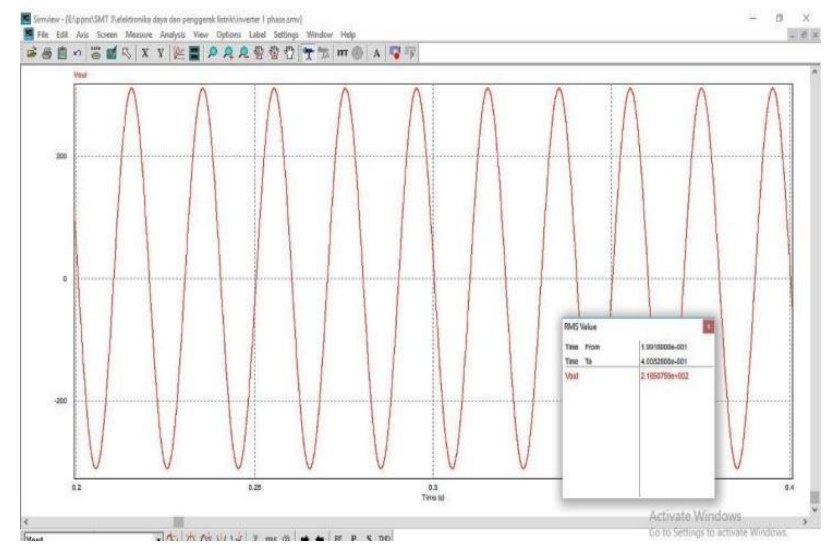

V. RESULT AND DISCUSSION

Overall, the important stages of sine wave generation with the Sinusoidal Bipolar Pulse Width Modulation (SPWM) technique can be illustrated in Figure 12.

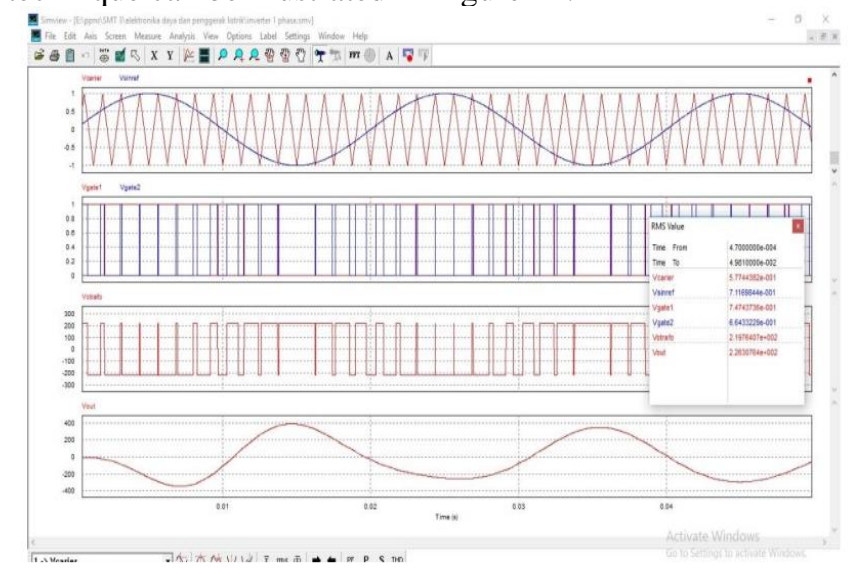

The following is a description of how the overall circuit is simulated with the PSIM Power Simulator software.

a. Batteries supply DC voltage which is later converted to AC voltage

b. The SPWM wave generator circuit that produces variations in pulse width as a trigger for mosfet switches on a fullbridge inverter circuit.

c. SPWM As a producer of voltage pulses used to drive mosfets with a duty cycle that varies according to variations in SPWM pulses.

d. Mosfet as a switch, was chosen because it has a fast switching time.

e. High frequency transformers are used to increase the signal amplitude to 220 Volts.

f. Capacitors and Inductors are used as low pass filters to pass only low frequencies below the cut-off frequency.

g. The output of the filter, in this simulation has become a sinusoidal AC voltage form.

The SPWM bipolar inverter system with analog SPWM generator simulated here can continue to be developed. For example by looking at how the system changes the type and value of the load. Feedback signals can also be added to improve output. And intelligent algorithms such as neural networks and meta-heuristics can be applied to get better control and output.

\section{REFERENCES}

[1] Mochamad Ashari, "Design of Power Electronics Converter", First Edition, Informatika Bandung, Bandung, 2017

[2] Francisco C. De La Rosa, "Harmonics And Power Systems, Distribution Control Systems", Inc., Taylor and Francis Group, Hazelwood, Missouri, USA, 2006.

[3] N.Mohan, T.M.Undeland, W.P.Robbins, "Power electronicsconverters, applications and design", Second edition, John Wiley \& Sons Inc., 1995

[4] Mr. V. M. Deshmukh, Ms. V. L. Jadhav, 2014, Comparative Analysis for Power Electronic Converter, International Journal Of Engineering Research \& Technology (IJERT) Volume 03, Issue 12 (December 2014),

[5] Muhammad H .Rashid "Power Electronics circuits, Devices and Applications "Third Edition, Sixth Impression,2009 